\title{
Spontaneous late improvement of myocardial viability in the chronic infarct zone is possible, depending on persistent TIMI 3 flow and a low grade stenosis of the infarct artery
}

\author{
M Faraggi, G Montalescot, L Sarda, J F Heintz, D Doumit, G Drobinski, I Sotirov, \\ D Le Guludec, D Thomas
}

\begin{abstract}
Objective-In the chronic phase of myocardial infarction, the relation between myocardial recovery and infarct related artery status remains unclear. The spontaneous changes in rest-redistribution thallium defect size were prospectively studied over six months in 52 patients with chronic $Q$ wave myocardial infarction.

Design-Changes in rest thallium defect size, thallium uptake in the infarct area, and radionuclide left ventricular ejection fraction were compared to the quantitative coronary angiogram data. Two groups of patients were considered: patients with a percentage of stenosis below $100 \%$ (group 1, $n=31$ ); and patients with an occluded artery (group $2, \mathbf{n}=21$ ).

Results-In the overall population, the mean (SD) defect size decreased from 28.2 $(17.2) \%$ to $24.9(19.3) \%$ of the whole myocardium $(p=0.01)$, while, in this area, the thallium uptake increased from $62.9(13.7) \%$ to $66.9(15.6) \%(p<0.001)$. At the time of inclusion, the defect size, thallium uptake, and ejection fraction were similar in both groups. In group 1 patients only, the reduction in defect size correlated with the improvement in ejection fraction $(r=0.41, p=0.02)$ and was related to the percentage of coronary artery stenosis. TIMI 3 patients reduced the defect size while other patients increased this defect $(-5.1(7.0) \% v+11.0(14.4) \%$, $p<0.001)$. In contrast, no significant relations were found in group 2 patients.

Conclusion-Late spontaneous recovery in thallium defect can occur in patients with a patent infarct related artery, depending on the TIMI flow grade and a low grade stenosis of the infarct related artery, and is associated with functional improvement.

(Heart 1999;81:424-430)
\end{abstract}

Keywords: myocardial infarction; thallium; infarct related artery; TIMI 3 flow grade

Coronary reperfusion of acute myocardial infarction improves survival ${ }^{1}$ and induces myocardial salvage, as confirmed by the decrease in the presumed size of the infarct area evaluated by serial sestamibi ${ }^{2}{ }^{3}$ or thallium ${ }^{4}$ scintigraphy.
During the hospital phase, the infarct related artery patency and TIMI (thrombolysis in myocardial infarction) perfusion grade 3 at discharge are among the best prognostic indicators of survival. ${ }^{5}$

After discharge, the initial in-hospital benefit of thrombolysis is sustained throughout five years without additional late improvement of mortality rate, ${ }^{5}$ suggesting that the major determinants for prognosis are collected during the hospital phase. However, some late cardiac events may impair the initial benefit of reperfusion on global and segmental left ventricular function. ${ }^{6}$ After successful thrombolysis, reocclusion is observed in about 30\% of patients within three months; patients with an occluded infarct related vessel have a lower global left ventricular ejection fraction and greater impairment in regional contractility than patients with a patent artery. ${ }^{46}$ Although the patency of the infarct related artery is a strong predictive factor, investigations performed during the acute phase underestimate myocardial salvage. Akinetic areas may contain viable but chronically hypoperfused myocardium, ${ }^{78}$ which can be detected by rest thallium myocardial scintigraphy, ${ }^{9-11}$ and baseline wall motion studies are not sufficiently accurate to evaluate the beneficial effects of reperfusion. ${ }^{12}{ }^{13}$ Delayed recovery of global and regional left ventricular function can occur within six months following discharge either after coronary revascularisation or spontaneously. ${ }^{14}$

Therefore, although they are major determinants for late survival, vessel patency and TIMI perfusion grade assessed within the first days following an acute myocardial infarction do not explain all the changes in myocardial functional or metabolic conditions occurring during the months after discharge. In this chronic phase of coronary artery disease, the relations between scintigraphic estimation of infarct size and viability, late coronary artery status, and recovery of left ventricular function remain unclear. Our study aimed to evaluate prospectively the spontaneous changes in the infarct area, as assessed by rest thallium tomoscintigraphy, with regard to vessel patency, coronary flow grade, and residual stenosis in patients enrolled late after a chronic $Q$ wave myocardial infarction. 


\section{Methods}

POPULATION

Patients with a $\mathrm{Q}$ wave myocardial infarction more than three weeks previously were included consecutively, whatever the treatment received during the acute phase (for example, whether they were reperfused or not). The diagnosis of myocardial infarction was supported by a typical history of chest pain and abnormal Q waves (>30 ms) in at least two contiguous leads, and by increased creatine kinase concentrations above twice the upper normal values. Patients who had experienced a recent (less than three weeks) myocardial infarction, those with postmyocardial infarction, unstable angina, or low threshold ischaemia not controlled by medical treatment, those who had undergone previous coronary bypass surgery, and those with supraventricular arrhythmias, were excluded. Patients who underwent coronary angioplasty before inclusion were eligible for the study but patients who needed coronary revascularisation or who had a change of their medical treatment during the six month follow up period were excluded. All patients gave their informed consent to the study.

\section{STUDY DESIGN}

All patients underwent coronary angiography and rest-redistribution thallium tomoscintigraphy within the same week for assessment of coronary artery status, residual viability, and myocardial scar. Left ventricular ejection fraction was determined by radionuclide angiography. Thallium tomoscintigraphy and radionuclide angiography were repeated six months later.

We evaluated the changes in the size of the thallium defects measured on redistribution images over the six month study period. The relations between these changes, the changes in left ventricular function, the percentage of stenosis, and the TIMI flow grade of the infarct related coronary artery were studied. In patients who had no percutaneous transluminal coronary angioplasty procedure, data concerning the infarct related coronary artery patency, TIMI grade flow, and coronary stenosis were collected on the coronary angiography performed just before the first thallium tomoscintigraphy. In patients who underwent coronary angioplasty before inclusion, coronary angiography was systematically repeated at the end of the six month follow up period to evaluate restenosis. The percentage of coronary artery stenosis and the TIMI flow grade considered for the study were those measured on this control angiography.

QUANTITATIVE CORONARY ANGIOGRAPHY

All cineangiograms were analysed with a computer assisted technique (Sigma Cardio, Traitement Synthèse Image, Paris, France) as previously reported, ${ }^{15}$ and measurements were made with no knowledge of the clinical or scintigraphic status. Briefly, the image on angiography was chosen to show the most severe incidence, at end diastole, with care taken to display the long axis of the vessel without overlapping and foreshortening. Each cine frame was digitised by a high resolution video camera, and the image was magnified. The region of interest centred on the stenosis was drawn, and vessel contours were automatically determined on the basis of the arterial centre line. The operator placed cross sectional lines at stenosis and reference locations, and the computer automatically calculated artery diameters along these lines. The computer measured the absolute values of the stenosis and reference diameters using the known catheter diameter as a scaling device. Because the algorithm was not able to measure total occlusions, a value of $0 \mathrm{~mm}$ for the minimal lumen diameter ( $100 \%$ stenosis) was given. ${ }^{15}$

\section{REST-REDISTRIBUTION THALLIUM} TOMOSCINTIGRAPHY

The first rest thallium acquisition was performed in the week following the patient's inclusion and started 10 minutes after intravenous administration of $111-148 \mathrm{MBq}$ (3$4 \mathrm{mCi}$ ) of ${ }^{201} \mathrm{Tl}$ according to the patient's bodyweight, with a rotating gamma camera (Elscint 409, Haiffa, Israel) fitted with a high resolution collimator. Acquisition was repeated 4 hours later for redistribution imaging. The same protocol was applied six months later, leading again to 10 minute postinjection and redistribution images.

Acquisition parameters were: $180^{\circ}$ circular orbit, starting with the right anterior oblique $30^{\circ}$ view in a supine position, $3064 \times 64$ matrix projections in a step and shoot mode (30 seconds per projection for rest and $40 \mathrm{sec}-$ onds per projection for redistribution). Slices were obtained by filtered back projection (Hamming-Hann filter) and reoriented along the three axes with a dedicated computer device (Sophy, SMV Sopha Medical, Buc, France). Background subtraction and attenuation correction were not used. A two dimensional mapping of tomographic data was automatically performed using a three dimensional radial sampling. A bull's eye polar map of the maximal value of the myocardial pixels (count rate density) was therefore displayed to compare rest and redistribution slices. ${ }^{16}$ Briefly, in this algorithm, the sampling is wholly angular and directly performed in three dimensional polar coordinates. The sampling vectors are distributed into a three dimensional cone including the whole myocardium. First, for each bull's eye polar map, a $60 \%$ level isocontour of the maximal value was generated. This isocontour perfectly delineated the area visually estimated as having a normal uptake. This isocontour level divided the bull's eye polar map into two regions of interest as previously described ${ }^{17}{ }^{18}$ : the area of normal thallium uptake and the thallium defect (expressed in percentage of the total area of the bull's eye). Since processing is fully automatic, this ensured maximal reproducibility in the computed values. For each polar map, a pathological/normal count ratio expressed as the ratio of the average values 
Table 1 Comparison of baseline characteristics of patients in groups 1 and 2

\begin{tabular}{llll}
\hline & $\begin{array}{c}\text { Group } 1 \\
(n=31)\end{array}$ & $\begin{array}{l}\text { Group } 2 \\
(n=21)\end{array}$ & p value \\
\hline Male/female & $26 / 5$ & $18 / 3$ & $0.8, \mathrm{NS}$ \\
Mean (SD) age (years) & $54(11)$ & $60(10)$ & 0.054 \\
Anterior myocardial infarction (ECG) & 17 & 10 & $0.6, \mathrm{NS}$ \\
Mean (SD) age of myocardial infarction (weeks) & $4(11)$ & $7(19)$ & $0.38, \mathrm{NS}$ \\
Number of vessels (1/2/3) (n) & $18 / 12 / 1$ & $9 / 9 / 3$ & $\mathrm{NA}$ \\
Infarct related artery (n) & & & \\
$\quad$ LAD & 7 & 10 & $\mathrm{NS}$ \\
LCx & 7 & 4 & $\mathrm{NS}$ \\
RCA & $51(18)$ & $46(17)$ & $\mathrm{NS}$ \\
Mean (SD) baseline LVEF (\%) & $26(18)$ & $31(16)$ & $0.25, \mathrm{NS}$ \\
Mean (SD) initial Tl defect size (\%) & & &
\end{tabular}

LAD, left anterior descending coronary artery; LCx, left circumflex coronary artery; RCA, righ coronary artery; LVEF, left ventricular ejection fraction; NA, not available; NS, not significant.

contained in the pixels of the defect area to the average values contained in the pixel of the normal area was automatically computed. This ratio was used as an index of the thallium uptake in the infarct area. As the extent of the thallium defect has been demonstrated to be a strong prognostic factor, ${ }^{19}$ it was considered the major studied variable.

RADIONUCLIDE ANGIOGRAPHY

After in vivo erythrocyte labelling with $1110 \mathrm{Mbq}(30 \mathrm{mCi})$ of $99 \mathrm{~m}$ technetium, gated equilibrium radionuclide studies were acquired in the left anterior oblique projection with the best septal visualisation (between $30^{\circ}$ and $40^{\circ}$ ) after the completion of redistribution thallium acquisition. Sixteen images $(64 \times 64$ matrix, zoom value of 2.0 ) per cardiac cycle were acquired. Gating was triggered by the ECG R wave, with a window of $20 \%$ centred on the average cardiac cycle length until a total count of $400 \mathrm{Kc}$ per image was achieved. Acquisition was computed on the same dedicated computer as thallium data using a standard software package automatically delineating two regions of interest around the left ventricle. Regions of interest may be manually corrected with the help of Laplacian, amplitude, or phase images. Using this software, normal left ventricular ejection fraction values ranged between 0.55 and 0.75 , and interobserver reproducibility was $r=0.97$.

STATISTICAL MANAGEMENT

All values were expressed as mean (SD). Quantitative comparisons were performed using either paired or unpaired Student's $t$ test when appropriate. Association between quantitative variables was determined by single regression analysis, using the Statistica IBM PC software. Association between qualitative variables was determined by $\chi^{2}$ test; $p \leqslant 0.05$ was considered significant.

\section{Results}

POPULATION

Fifty two consecutive patients (44 men, eight women, age 56 (11) years) were included in this study. Four patients had a prior myocardial infarction in another area than the one studied, and 10 had residual exertional chest pain. Based on ECG findings, the myocardial infarction was anterior in 27 patients, inferior in 22 , and lateral in three patients. Inclusion occurred
5 (15) months (range 3 weeks to 72 months) after the acute phase, six patients having a myocardial infarction older than six months. In the acute phase, 23 patients received thrombolytic treatment and two more patients underwent rescue angioplasty. Before inclusion in the study, 29 patients had delayed coronary angioplasty including 26 patients with coronary angioplasty of the infarct related artery, which was performed 4 (1.8) weeks after the acute phase. Ongoing drug treatments were maintained throughout the study.

\section{CATHETERISATION DATA}

Twenty seven patients had one vessel disease, 21 patients had two vessel disease, and four patients had three vessel disease. The infarct related artery was the left anterior descending coronary artery in 27 patients, the left circumflex artery in 11 patients and the right coronary artery in 14 patients. According to the stenosis of the infarct related artery, patients were divided in two groups (table 1). Group 1 patients had a non-occluded infarct related artery (coronary artery stenosis 0-99\%, $\mathrm{n}=31$ ) and group 2 patients had an occluded coronary artery (coronary artery stenosis $100 \%, n=21$ ). For both groups, the thallium uptake in the infarct area and the size of the thallium defect were not significantly different when measured on postinjection images or on redistribution images on inclusion, as well as after the follow up period. Therefore, for all further comparisons, we retained only data obtained with the redistribution images.

At the time of inclusion, the age of myocardial infarction, the initial left ventricular ejection fraction, the defect size, or the thallium uptake in the infarct area were not significantly different between the two groups. Group 1 patients were slightly older than those in group 2 (59.6 (9.8) years $v 53.8$ (10.6) years, $\mathrm{p}=0.054)$. In group 1 patients, the average stenosis of the infarct related artery was 43 (17)\% (range 12-75\%); 27 patients had a TIMI 3, three had a TIMI 2, and one a TIMI 1 grade coronary flow. The 21 patients in group 2 had a TIMI 0 grade flow. All 26 patients who underwent coronary angioplasty before inclusion in the study had a control coronary angiogram at six months' follow up, and the second thallium tomography within the same week. In these patients, we considered the stenosis measured on the second angiography performed at six months took into account the restenosis process.

EVOLUTION OF THALLIUM DEFECT AND LEFT VENTRICULAR EJECTION FRACTION WITHIN THE SIX MONTHS

For the overall population, the size of the infarct area as evaluated by redistribution thallium scans decreased spontaneously from 28.2 (17.2)\% of the whole bull's eye on inclusion to $24.9(19.3) \%$ at six months $(p=0.01)$, while thallium uptake in this area increased from $62.9(13.7) \%$ to $66.9(15.6) \%(p=0.0003)$. Comparing the two groups of patients, the reduction in thallium defect size (group $1-4.5$ 


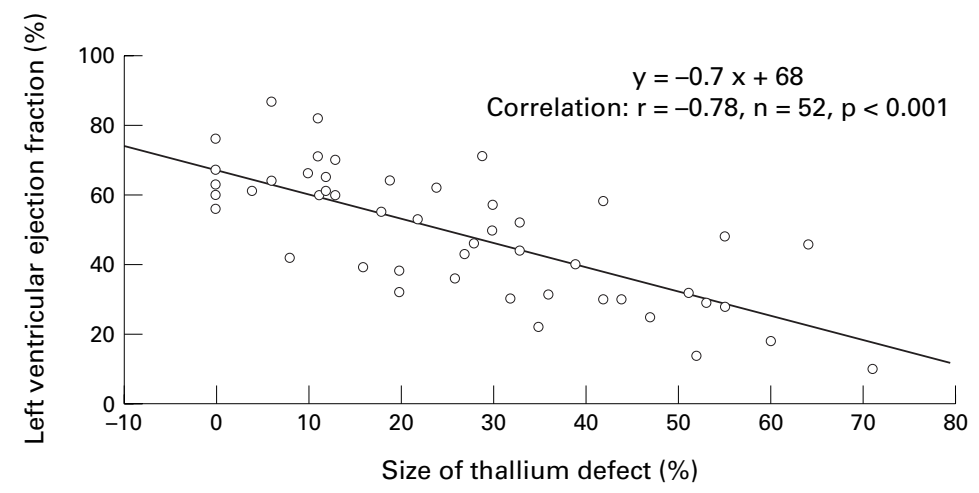

Figure 1 Correlation between thallium defect size (measured on redistribution images at six months) and global left ventricular ejection fraction.

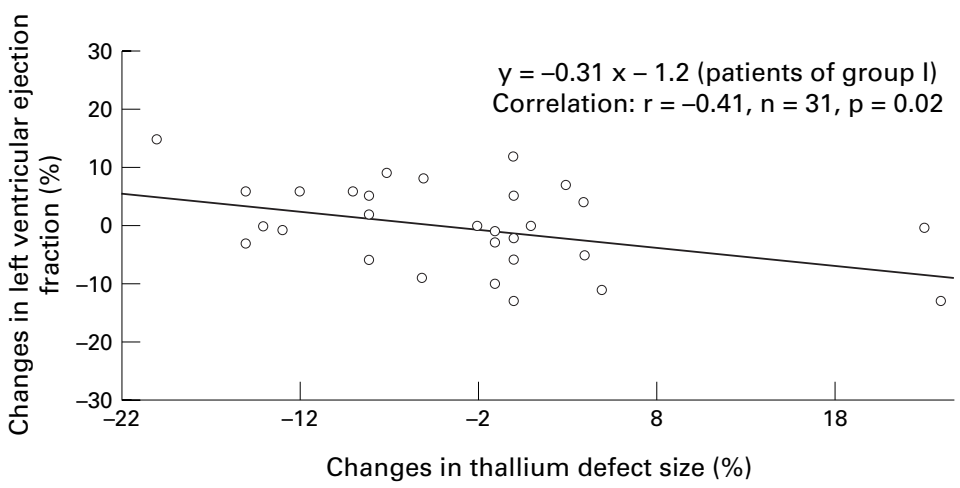

Figure 2 Correlation between the changes in left ventricular ejection fraction and the changes in thallium defect size (on redistribution images) in patients with coronary stenosis of the infarct related artery below 100\%. The difference between data obtained on inclusion and data collected at six months were plotted.

$(7.9) \% \quad v$ group $2-3.7(7.5) \%$ ) and the improvement of thallium uptake in the infarct area (group $1+3.6$ (8) $\%$ v group $2+3.0$ $(9.7) \%)$ did not differ significantly.

No significant change in left ventricular ejection fraction was found in the whole population at six months (from 49.1 (18.0)\% to 49.8 $(17.9) \%$, NS). There was no difference between groups for the number of patients improving their left ventricular ejection fraction (group 1 $12 / 31(39 \%) v$ group $211 / 21$ (52\%), NS).

On inclusion, the size of the thallium defect was correlated to ejection fraction $(r=-0.67$, $\mathrm{p}<0.001, \mathrm{n}=52)$. At six months, this correlation was closer $(r=-0.78, \mathrm{p}<0.001, \mathrm{n}=52)$ (fig 1).

Within the six months, the individual reduction in the size of the thallium defect was significantly related to the individual improvement in left ventricular ejection fraction in

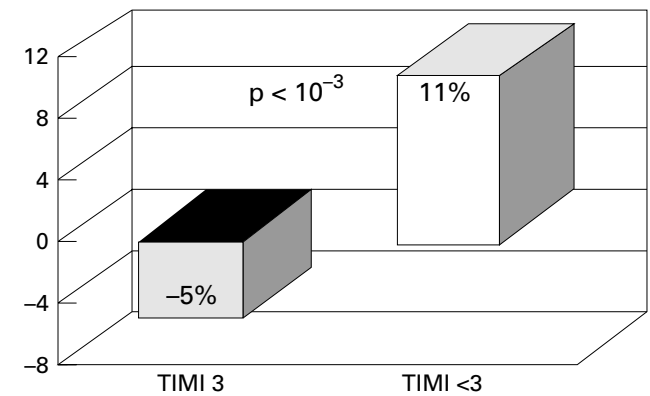

Figure 3 Changes in thallium defect size (difference between the defect size on inclusion and the defect size at six months) in group 1 patients according to TIMI flow grade. group 1 patients only $(r=-0.41, \mathrm{p}=0.02)$ (fig 2). This relation was not found in patients with an occluded artery (group $2 r=0.08$, $\mathrm{p}=0.7)$. In group 1 , patients with $>5 \%$ reduction in thallium defect size had a significantly increased ejection fraction compared to patients with a $<5 \%$ reduction or with a worsening in this defect size (change in ejection fraction $+3.3(5.7) \%, \mathrm{n}=12, v-2.5$ $(7.4) \%, n=19, p=0.03)$. Conversely, eight of 21 patients in group 2 reduced the infarcted area above $5 \%$ but without significant changes in left ventricular ejection fraction. No relation was found between this reduction in infarct size and the Rentrop evaluation of coronary collateral flow.

Therefore, the relation between improvement in thallium uptake and improvement in left ventricular function affected only patients with a patent infarct related coronary artery.

RELATION BETWEEN SCINTIGRAPHIC EVALUATION OF THE INFARCT AREA AND CORONARY ANGIOGRAPHY DATA

In group 1 patients, a significant inverse relation was found between the percentage of coronary artery stenosis and the reduction in the size of redistribution thallium defect $(r=-0.37, \mathrm{p}=0.04, \mathrm{n}=31)$ (fig 2$)$, particularly for patients with anterior myocardial infarction $(r=-0.51, \mathrm{p}=0.04, \mathrm{n}=17)$. TIMI 3 patients reduced their defect size while the others increased this defect size (changes -5.1 $(7.0) \% v+11.0(14.4) \%, p<0.001)$ (figs $3-5)$. Conversely, no significant improvement in the size of the redistribution thallium defect was found in patients who underwent coronary angioplasty compared to those who did not, or in patients who had experienced a recent myocardial infarction (less than four weeks previously) compared to patients whose myocardial infarction had occurred more than four weeks previously.

\section{Discussion}

The main findings of our study are: (1) late after myocardial infarction, a significant reduction of the rest thallium defect and an increase of thallium uptake within the infarct area can occur spontaneously, even in patients with an occluded infarct related artery; (2) despite the number of patients in our study whose spontaneously improved left ventricular ejection fraction was not different in regard to patent or occluded infarct related artery, we observed a significant relation between this reduction in thallium defect size and an increase in global left ventricular function only in patients with a patent artery; and (3) in this subset of patients, the improvement in defect size was related to the degree of coronary artery stenosis and to TIMI 3 flow grade, especially in patients with an anterior myocardial infarction.

The time course and the mechanisms of recovery of left ventricular function after myocardial infarction remain unclear. Previous studies demonstrated that most improvement occurs during the hospital phase and is 


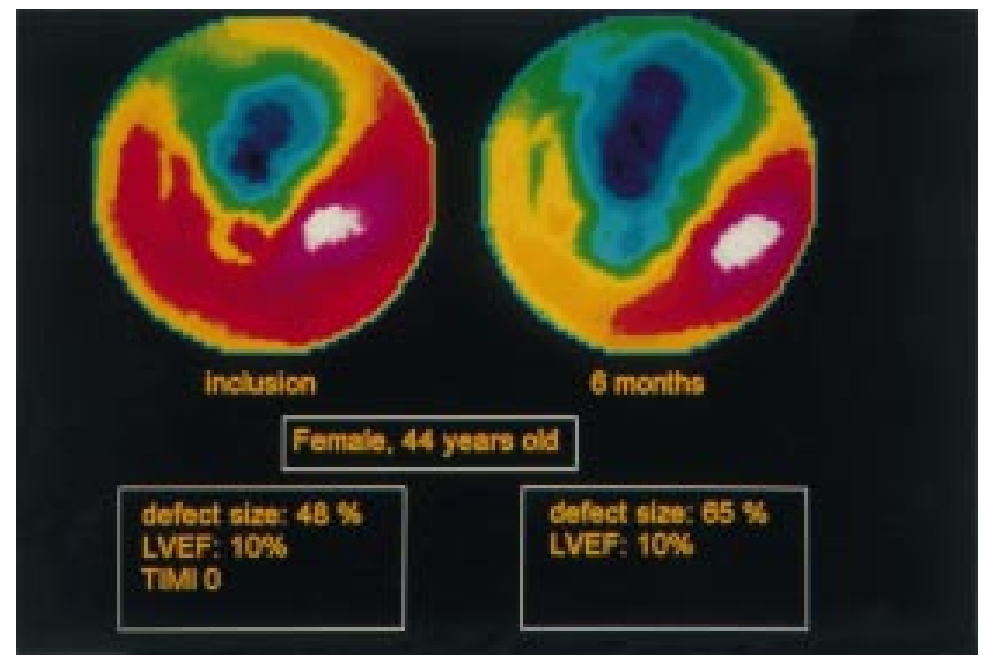

Figure 4 Forty four year old woman after anterior myocardial infarction and TIMI 1 flow grade of the left anterior descending artery. Bull's eye polar map on inclusion (left) and six months later (right) demonstrating the worsening of the thallium defect on the septal wall. LVEF, left ventricular ejection fraction.

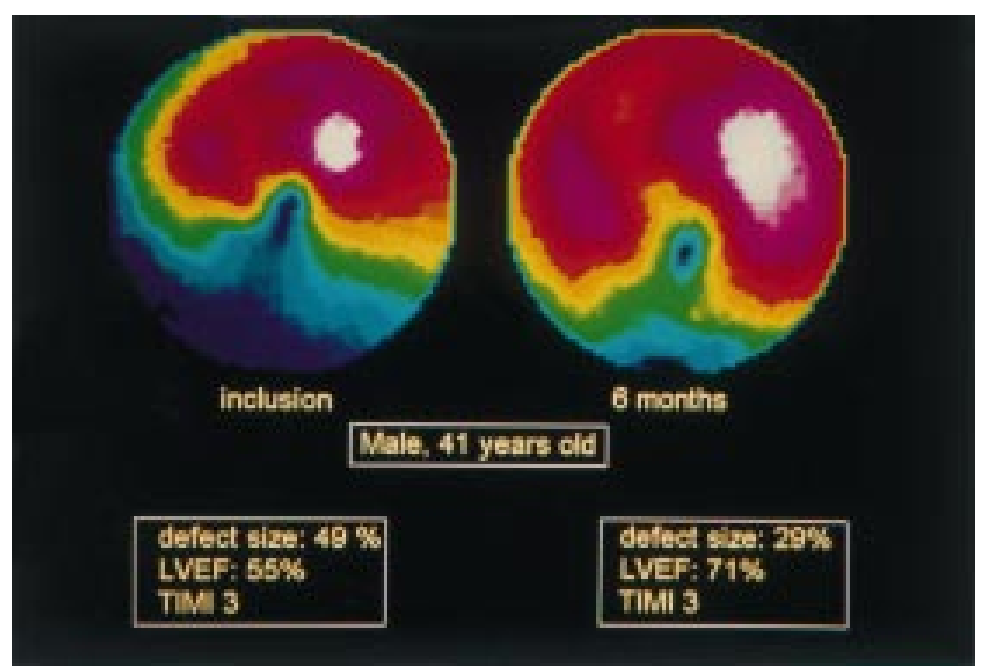

Figure 5 Forty one year old man after inferior myocardial infarction and TIMI 3 flow grade of the right coronary artery. Bull's eye polar map on inclusion (left) demonstrating an inferior thallium defect involving the contiguous septal and lateral walls. Six months later (right), improvement of the thallium uptake in the bordering areas of the inferior defect. LVEF, left ventricular ejection fraction.

related to early TIMI 3 flow in the infarct related coronary artery. ${ }^{5}$ This supports the "open artery theory", ${ }^{20}$ which stipulates that a preserved coronary flow in the area at risk during the hospital phase is beneficial in terms of cardiac events and left ventricular function. Nevertheless, the relation between infarct related vessel status and myocardial function in the chronic phase of the disease remains controversial. Some late recovery (after discharge) in left ventricular function may occur either spontaneously ${ }^{14}$ or after percutaneous transluminal coronary angioplasty, even in the absence of documented myocardial ischemia. ${ }^{17}$ This improvement is also related to an improvement in sestamibi ${ }^{14}$ or thallium ${ }^{17}$ uptake. In patients with a totally occluded artery, subjects in whom myocardial contrast echocardiography showed major residual perfusion improved their left ventricular function after delayed reperfusion, even weeks after infarction. ${ }^{21}$ The mechanisms of this late recovery remain unclear and probably involve the concepts of stunned ${ }^{22}$ or hibernating myocardium in case of persistent functionally significant coronary stenosis. $^{78}$ Vessel patency, coronary flow grade, or severity of coronary artery stenosis were not clearly related to this delayed improvement, ${ }^{14}$ but this latter study was not designed to address this point.

Our results appear to be concordant with those previously reported, since changes in defect size and thallium uptake within the infarct area did not relate to infarct related artery status when considering patients with or without an occluded artery. Conversely, if we considered separately the results of each group, functional recovery was inversely related to residual stenosis in patients with a patent artery. Moreover, reduction in defect size was obtained in TIMI 3 patients only, with a concomitant improvement of left ventricular function. This suggests that a sustained adequate coronary flow in the chronic phase of myocardial infarction and a low residual stenosis are beneficial in patients with a patent infarct related artery. Some patients with an occluded infarct related artery may also reduce their defect size by more than $5 \%$, but this improvement is not correlated to a functional improvement. This uncoupling between thallium uptake and regional function confirms that the functional recovery requires a higher perfusion threshold than the persistence of a myocardial metabolism, as previously suggested. ${ }^{23}$ Therefore, our data suggest that, in the chronic phase of myocardial infarction, myocardial viability may be persistent for a long time, even when the infarct related artery is occluded, but a preserved antegrade coronary flow is required to improve left ventricular function. However, during the six month observation period, the changes either in thallium uptake or in ejection fraction are mild and individual mismatch between both parameters may occur, despite the correlation remaining significant. Surprisingly, and despite the fact that the difference was not significant, $39 \%$ of patients with a patent infarct related vessel and $52 \%$ of patients with an occluded artery improved left ventricular ejection fraction. Since patients are included at least three weeks after the acute phase, most of the functional recovery may have already occurred in patients with patent vessel and low grade stenosis of the infarct artery. Therefore, functional improvement could be underestimated during the study period in group 1 patients, minimising the differences in left ventricular function and thallium uptake improvement between both groups.

The present study differs from others on several points. Our patients were included late after the acute phase, suggesting that the recovery of function or tracer uptake can occur long after the acute event. Rest-redistribution thallium has been demonstrated as an accurate tool to study myocardial viability. ${ }^{10}{ }^{24}$ After myocardial infarction, thallium uptake is inversely correlated with myocardial fibrosis, ${ }^{25}$ 
even in the presence of a flow limiting residual coronary stenosis. ${ }^{26-28}$ Furthermore, thallium uptake is related to functional recovery, ${ }^{24} 28$ even if the degree of cellular myocyte integrity required for functional recovery and for thallium uptake may be different. Compared to these studies, mean thallium uptake in the infarct area of our patients (at the time of inclusion) was rather high (63\%) and concordant with the persistence of myocardial viability. The changes in left ventricular function seem too mild to relate the increase in thallium uptake to a greater degree of wall motion abnormality on baseline and to partial volume effects.

Several limitations of the present study deserve further consideration. Inclusion of patients and comparison between scintigraphic and catheterisation data were prospective, but the examination of the impact of thrombolysis or coronary angioplasty in further improvement was retrospective and not randomised. However, the primary goal of our study was not to demonstrate the efficacy of coronary reperfusion or revascularisation but to assess, in the chronic phase of myocardial infarction, the relation between infarct related artery status and late evolution of thallium defect size. Left ventricular remodelling was not directly evaluated despite the fact that left ventricular enlargement and/or aneurysm formation are known to decrease left ventricular function. However, in our patients with chronic Q wave infarction, we observed a reduction rather than an extension of infarct size and a significant link between this reduction in infarct size and left ventricular function improvement in patients with patent infarct related artery.

Our results support the proposal that the presence of an open infarct related artery, a preserved coronary flow, and no residual stenosis could be beneficial when still present late after the acute phase. This raises several questions about patient management following myocardial infarction. Firstly, before discharge or during the subacute phase, myocardial salvage and left ventricular function evaluations may be underestimated, especially if the infarct related artery is patent with a preserved coronary flow. Secondly, coronary revascularisation may have an important impact in patients with a patent artery but a tight stenosis to restore part of the contractile function, confirming previous studies. ${ }^{11}{ }^{172324}$ Thirdly, in the particular group of patients with an occluded artery, the evidence of myocardial viability with thallium scintigraphy is not able to predict alone the spontaneous changes in global ejection fraction.

CONCLUSION

After a $\mathrm{Q}$ wave myocardial infarction, the size of rest thallium defect may decrease and thallium uptake within the infarct area may improve spontaneously, even when patients are investigated late after the acute phase. This reduction in thallium defect size is related to an increase in global left ventricular function in patients with a patent infarct related artery. In this subset of patients, the improvement in defect size is related to the degree of coronary artery stenosis and to TIMI 3 flow grade.

1 Simoons ML, Serruys PW, Van den Brand M, et al. Early thrombolysis in acute myocardial infarction: limitation of infarct size and improved survival. $f$ Am Coll Cardiol infarct size and

2 Clements IP, Christian TF, Higano ST, et al. Residual flow to the infarct zone as a determinant of infarct size after direct angioplasty. Circulation 1993;88:1527-33.

3 Pellika PA, Behrenbeck T, Verani MS, et al. Serial changes in myocardial perfusion using tomographic Tc $99 \mathrm{~m}$ hexakis-2methoxy-2-methylpropyl-isonitrile imaging following reperfusion therapy of myocardial infarction. $f$ Nucl Med 1990;31:1269-75.

4 Hirayama A, Adachi T, Asada S, et al. Late reperfusion of acute myocardial infarction limits the dilation of left ventricle without the reduction of infarct size. Circulation 1993;88:2565-74.

5 Lenderink T, Simoons ML, Van Es GA, et al, for the European Cooperative Study Group. Benefit of thrombolytic therapy is sustained throughout five years and is related to TIMI perfusion grade 3 but not grade 2 flow at discharge. Circulation 1995;92:1110-16.

6 Meijer AM, Verheugt FWA, van Eenige MJ, et al. Left ventricular function at 3 months after successful thrombolysis: impact of reocclusion without reinfarction on ejection fraction, regional function and remodeling. Circulation 1994; 90:1706-14

7 Rahimtoola SH. The hibernating myocardium. Am Heart $f$ 1989;117:211-21.

8 Braunwald E. Myocardial reperfusion, limitation of infarct size, reduction of left ventricular dysfunction and improved survival: should the paradigm be expanded? Circulation 1989;79:441-4

9 Bonow RO, Dilsizian V, Cuocolo A, et al. Identification of viable myocardium in patients with chronic coronary artery disease and left ventricular dysfunction: comparison of thallium scintigraphy with reinjection and PET imaging with ${ }^{18} \mathrm{~F}$-fluorodeoxyglucose. Circulation 1991;83:26-37.

10 Dilsizian V, Perrone-Filardi P, Arrighi JA, et al. Concordance and discordance between stress-redistributionreinjection and rest-redistribution thallium imaging for assessing viable myocardium: comparison with metabolic assessing viable myocardium: comparison with metabolic activity by

11 Ragosta M, Beller GA, Watson DD, et al. Quantitative planar rest-redistribution 201TL imaging in detection of myocardial viability and prediction of improvement in left ventricular function after coronary bypass in patients with severely depressed left ventricular function. Circulation 1993;87:1630-41

12 Califf RM, Harrelson-Woodlief L, Topol EJ. Left ventricular ejection fraction may not be useful as an end point of thrombolytic therapy comparative trials. Circulation 1990; 82:1847-53.

13 Van de Worf F. Discrepancies between the effects of coronary reperfusion on survival and left ventricular function. Lancet $1989 ;$; 1 1367-9.

14 Galli M, Marcassa C, Bolli R, et al. Spontaneous delayed recovery of perfusion and contraction after the first 5 weeks recovery of perfusion and contraction after the first 5 weeks nating myocardium in the infarcted area. Circulation 1994; 90:1386-97.

15 Montalescot G, Ankri A, Vicaut E, et al. Fibrinogen after coronary angioplasty as a risk factor for restenosis. Circulaion 1995;92:31-8.

16 Goris ML, Boudier S, Briandet PA. Two dimensional mapping of three dimensional SPECT data: a preliminary step to the quantitation of thallium myocardial perfusion single photon emission tomography. Am $f$ Physiol Imaging 1987;2:176-80.

17 Montalescot G, Faraggi M, Drobinski G, et al. Myocardial viability in patients with $\mathrm{Q}$-wave myocardial infarction and no residual ischemia. Circulation 1992;86:47-55.

18 Steg PG, Faraggi M, Himbert D, et al. Comparison using dynamic vectorcardiography and MIBI-SPECT of ST segment changes and myocardial MIBI uptake during ment changes and myocardial MIBI uptake during percutaneous transluminal coronary angioplasty of the left $998-1002$.

19 Machecourt J, Longère $\mathrm{P}$, Fagret $\mathrm{D}$, et al. Prognostic value of thallium 201 single photon emission computed tomographic myocardial perfusion imaging according to extent of myocardial defect. $\mathcal{F}$ Am Coll Cardiol 1994;23:1096-106. 20 Ambrose JA. The open artery: beyond myocardial salvage. Am 7 Cardiol 1993;72:85G-90G.

21 Sabia PJ, Powers ER, Ragosta M. An association between collateral blood flow and myocardial viability in patients with recent myocardial infarction. $N$ Engl f Med 1992;327: $1825-31$.

22 Bolli R. Myocardial stunning in man. Circulation 1992;86: $1671-88$

23 Panza JA, Dilsizian V, Laurienzo JM, et al. Relation between thallium uptake and contractile response to dobutamine: implications regarding myocardial viability in patients with chronic coronary artery disease and left ventriculh chronic coronary artery disease and left ventricular
dysfunction. Circulation 1995;91:990-8. 4 Perrone-Filardi P, Pace L, Prastaro M, et al. Assessment of myocardial viability in patients with chronic coronary artery disease: rest 4 hour- 24 hour $\mathrm{Tl}$ tomography versus dobutamine echocardiography. Circulation 1996;94:271219. 
25 Zimmermann R, Mall G, Rauch B, et al. Residual ${ }^{201} \mathrm{Tl}$ activity in irreversible defects ity in irreversible defects as a marker of myocardial viability

26 Cuocolo A, Pace L, Ricciardelli B, et al. Identification of viable myocardium in patients with chronic artery disease: comparison of thallium 201 scintigraphy with reinjection and Tc99m methoxyisobutyl isonitrile. $f$ Nucl $\mathrm{Med}$ 1992;33:505-11.
27 Marzullo P, Parodi O, Reisenhofer B, et al. Value of rest thallium 201/technetium $99 \mathrm{~m}$ sestamibi scans and dobthallium $201 /$ technetium $99 \mathrm{~m}$ sestamibi scans and dobutamine echocardiography for det

28 Sawada GS, Allman KC, Muzik O, et al. Positron emission tomography detects evidence of myocardial viability in rest Tc99m sestamibi defects. F Am Coll Cardiol 1994; 23:92-8.

\section{IMAGES IN CARDIOLOGY}

\section{Acute myocardial infarction from paradoxical embolism in a case of massive pulmonary thromboembolism}

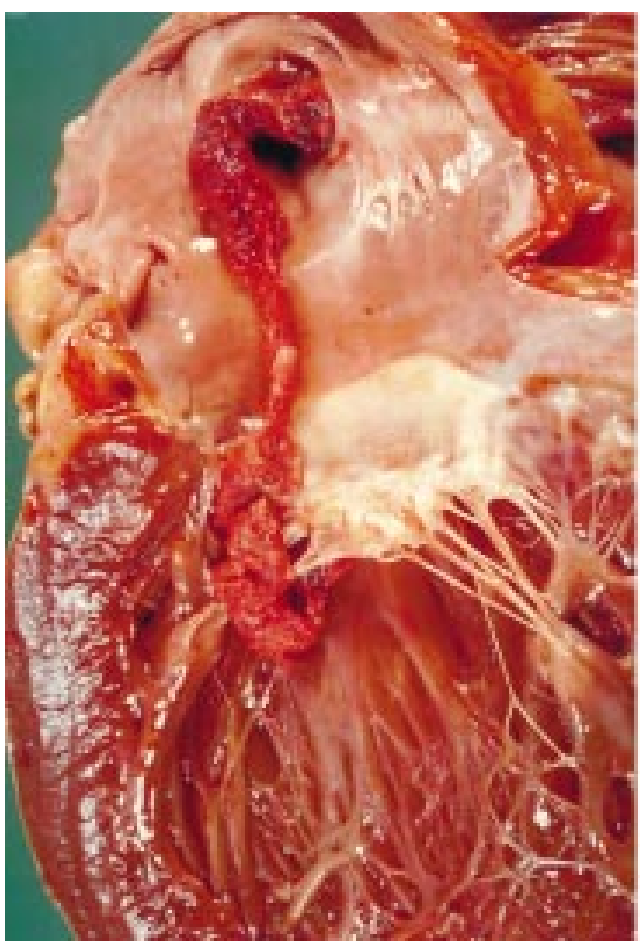

A 46 year old man was admitted to hospital because of a two month history of hip pain. Radiography of the leg was consistent with an aseptic necrosis of the femur. Ten days later the patient became acutely dyspnoeic and had signs of severe pulmonary hypertension. Echocardiography demonstrated hypomobility of the inferior vena cava and right atrium. The patient was treated with heparin and streptokinase but died towards the end of the procedure.

Post mortem examination revealed a massive, bilateral pulmonary thromboembolism. The heart was enlarged and weighed $415 \mathrm{~g}$. An atrial septal defect involving the region of the fossa ovalis was found. A long embolus reached

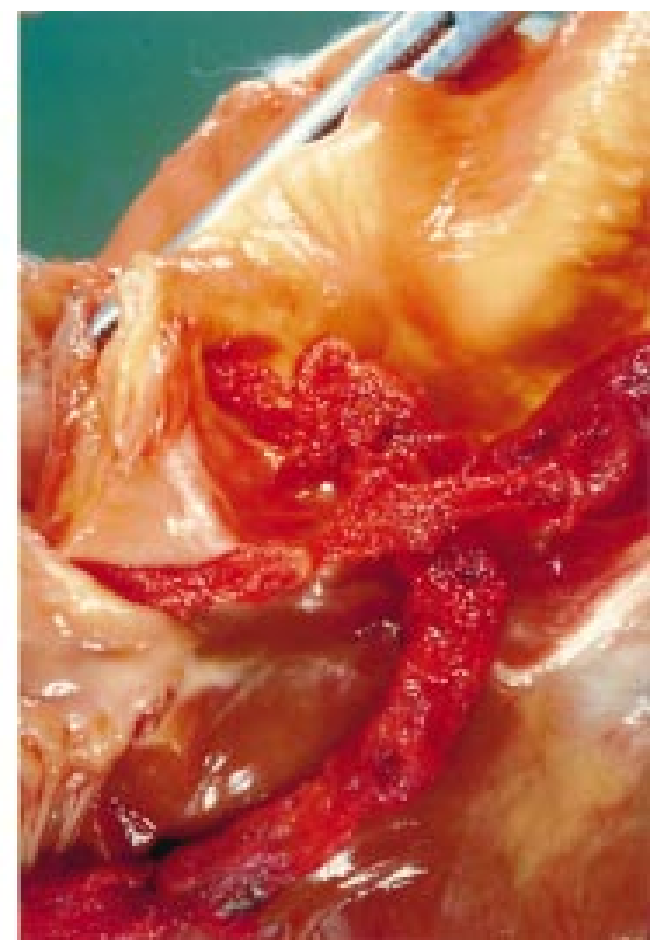

the right atrium, passed through the foramen ovalis of the atrial septum (left) and thence through the left ventricle to lodge in the circumflex left coronary artery (right). Multiple small infarctions were detected in the brain, spleen, and left kidney.

Histology showed an extensive subendocardial necrosis in the lateral wall of the left ventricle. The massive pulmonary arteries obstruction, causing a significant rise of right atrial pressure, allowed the right to left shunt of thromboembolic material through the atrial septal defect.

R BUSSANI N PAVLETIC F SILVESTRI 